\title{
Designs of the psychological assessment and test system based on high-level talent management
}

\author{
MENG Xian-jun ${ }^{1, a}$ \\ ${ }^{1}$ Xi'an fanyi university, Xi'an, China \\ Shuhua_y@126.com
}

Keywords: High-level personnel, Test Project, Emotion Stabilization, Evaluating System

\begin{abstract}
According to the needs of the society for the introduction of high-level talents, the selection platform for talents through a psychological test is built and a psychological assessment system is designed. By using the design routine of modular design, advanced sensing technology, real-time evaluation and standard analysis on the whole, the psychological assessment and test system is established by using the personal computer as a core for data processing and calculation. The system is able to realize multiple functions, such as emotional stability test, cognitive ability test and intelligence scale test, and provides references for the selection and psychological assessments of talents for important and special positions.
\end{abstract}

\section{Introduction}

Under the environment of competition for talents in the society, the psychological quality of the introduced and selected talents is the key to the use of talents. Psychological testing is not only the objective needs for situation development, but also a basic standard to measure whether a talent is competent at certain occupations[1][2]. Special populations generally work in various extreme environments, such as environments at high altitudes or with cold, hot, noise, toxic substances, radiation, acceleration and vibration, bringing great challenges for their comprehensive psychological quality. The aim is to comprehensively and accurately grasp the psychological characteristics and laws of talents and special populations with a stable emotion, enhance the pertinence and validity of work and facilitate the selection of high-quality talents. For this purpose, it is urgent to develop a digital assessment system that can accurately quantify the comprehensive psychological quality. Now, the following introduces the designed assessment system for emotional stability[3][4].

\section{Overall design of the system}

System structure. By utilizing the design routine of modular design, real-time evaluation and standard analysis, the assessment system of emotional stability is designed by using the personal computer as a core for data processing and calculation. The system mainly consists of test items and auxiliary functions. The test items include five functional test modules, i.e. emotional stability test, cognitive ability test, intelligence scale test and test paper examination. The auxiliary functions can provide infinite upgrade and are compatible with a variety of psychological tests. Moreover, they consist of users' data entry, data review, data maintenance, data printing and expert consultation[5]. Main functions. Emotional stability test: the system can be used to quantitatively analyze the emotional stability parameters of the testees objectively and accurately, which can provide references for selecting talents for important positions[6].

Cognitive ability test: based on the latest psychological research results, the system can comprehensively, accurately and quantitatively evaluate the cognitive ability of people through various test means.

Intelligence scale test: the intelligence scale test is used for testing learning ability and integrates the sub-tests with the same performance to divide into two sub-scales. They are verbal scale including common sense, comprehension, arithmetic, similarity, digit span and vocabulary, and performance 
scale consisting of digital symbols, image filling, building blocks, object assembly and picture arrangement. Furthermore, talents are tested according to the difficulty degrees of these items.

\section{System hardware}

Hardware structure. The modular design ideas and advanced sensing technology are used for hardware design of the assessment system. The hardware mainly comprises a circuit for single-chip microcontrollers and processors, a data acquisition circuit, a background sound generating circuit, an alarm indicating circuit and an USB communication interface circuit.

Unit circuits. Data acquisition circuit and other circuits. The data comprising hand shaking frequency, pulse and bulk resistance of testees are collected in real time by using a sensor to obtain the original data. The data are sent to the circuit for single-chip microcontrollers and processors through scanning circuits for pre-processing and then transformed into the regulated communication format. Then, the data in a standard format are transferred to the personal computer through the USB interface and finally analyzed and processed using the special assessment software system. The specific circuits are shown in Figure 1. The background sound generating circuit is utilized to simulate various working environment for people and special populations. It generates different background sounds, so as to stimulate the sense system of testees, thus detecting the psychological enduring capacity of testees in the real working environment.

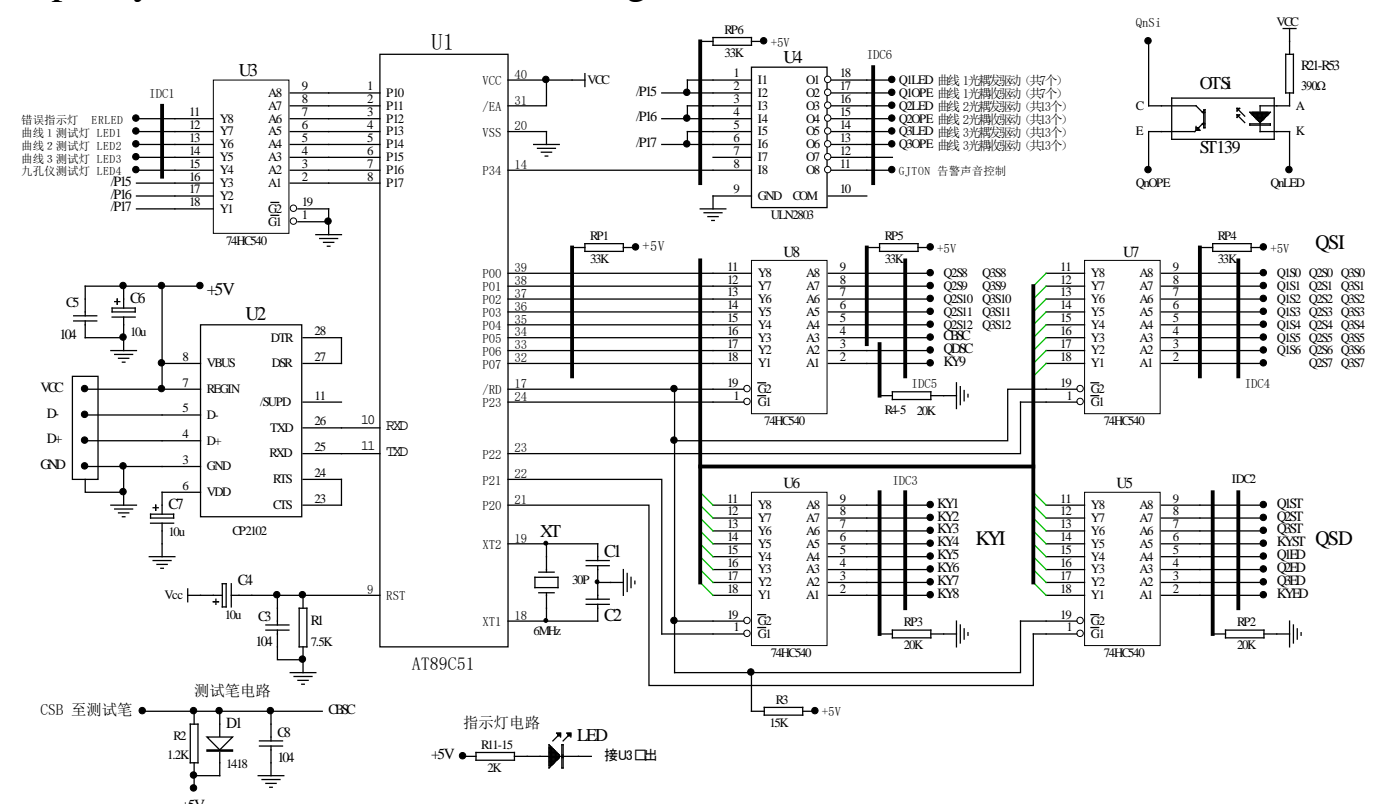

Figure1 Data acquisition circuit

The USB 2.0 interface showing a strong universality and a high transmission rate is used as the communication interface. The CP2101 used for interface chip is currently the strongest anti-jamming USB chip. In the test, when the operation mistakes occur, the testees are notified though the means, like light and sound.

Processor control circuit module. Processor control circuit module is the control center of the system and includes a digital storage, a CPU, a watchdog circuit and a clock circuit. The AT89C52 with $8 \mathrm{~K}$ of program storage and 256 bytes of data storage is used as the CPU. Due to a few external components, the CPU has extremely high operation reliability. The data storage mainly stores some temporary data, state information of selector switches and various alarm information in the processing. The watchdog is used to monitor the operation of the AT89C52 CPU. When the CPU program runs normally, the watchdog circuit is reset once at a specified time interval. Otherwise, the watchdog generates a hard reset signal for the single-chip microcontroller, so that the microcontroller can restore and work normally. 


\section{System software}

By using the object-oriented development environment, database, and network technology, the software is developed in a short cycle and various modules perform favorably. This makes the system more targeted and professional. The overall control can be divided into a menu bar, a basic data display bar, a shortcut button bar for test items and other miscellaneous controls.

Menu bar. In the menu bar, there are host nodes, child nodes, action and use occasions. The child nodes included in the host nodes for logging in the system consist of password login, adding administrators, deleting administrators, modifying administrators, administrator login, changing interface and exiting the system. The child nodes included in the host nodes for personnel setting comprise new user login, modifying users' information, deleting users' information and deleting users. The child nodes in the host nodes for checking and transferring comprise checking users' information, checking test results, turning to view page, data backup, exporting to DBF and exporting to EXCEL. These child nodes consisting of all print information, specific print information, print setup and performing print are included in the host nodes for printing. In addition, the child nodes in the host nodes for expert consultation are setting network, uploading test materials, logging in the Internet and online test paper.

Test items and operation flows. The implementation of all tests can continue to be filled. The main test items include emotional stability test, cognitive ability test, intelligence scale test and examination test. The test operation processes include the commonly used operations, like testing, viewing and printing, while excludes system login. The operation flow is shown as follows: selecting users to be tested, selecting test items, finishing, storing test results, checking and printing test results. Monitoring host communication programs. Multi-thread technology. There are a variety of thread synchronization methods, such as event objects, semaphores and mutexes. Event objects, as a core object, are used in this program and show the states including stimulation and non-stimulation. It is used in a thread to inform other threads the occurrence of a certain event and suits for the occasion that a thread cannot execute certain operations before an event occurs. Functions CreateEvent() and CloseHandle() are used to generate an event object and close the event object after utilization, respectively. Functions SetEvent() and ResetEvent() are utilized to set the event object as stimulated and non-stimulated one, respectively.

The WIN32 API provides the wait functions that can block the execution of threads and when the monitored objects generate specific signals or are in the specific state, the block stops and threads execute continuously. The design of the functions is to temporarily suspend threads to reduce the occupation of CPU resources and improve the efficiency of the program. The commonly used wait functions include WaitForSingleObject() and WaitForMultipleObjects(). The former can monitor a single synchronization object, while the latter can monitor multiple synchronization objects simultaneously.

Event driven and overlapping I/O mechanism. The system provides a high-level event-driven communication mechanism to encapsulate the underlying communication interrupts and provide a number of intuitive communication events to the application program, thus avoiding the direct operations on complex underlying hardware. By using the driven mechanism, the application program can obtain the occurrence mechanism of some conditions without checking port states. Particularly for receiving bytes, the application program does not need to continuously detect ports, thus saving CPU time.

By utilizing the event masks established through the function SetCommMask(), the application program can monitor the events in the specific serial port and then the event masks of this serial port can be obtained by using the function GetCommMask(). After specifying the communication events needed to be monitored using the function SetCommMask(), the application program can call the wait function WaitCommEvent() of communication events to wait for the occurrence of one of the communication events.

The function WaitCommEvent() can be used synchronously or asynchronously. When this function is used synchronously, if the waited communication events do not occur, this function will 
not return. When the serial ports are opened through the asynchronous mode, the function WaitCommEvent() should be utilized asynchronously. Otherwise, this function cannot correctly report the accomplishment of an operation. When this function is employed asynchronously, even if the waited communication events do not occur, this function will return immediately and the return value is FALSE. Moreover, the function GetLastError() returns with ERROR_IO_PENDING to indicate that the operation is executed in the background. At this time, before the function WaitCommEvent() returns, the system sets the event object hEvent in OVERLAPPED structure pointed by parameter lpOverlapped in the function to be non-stimulation state. When the waited communication events occur or have errors, the system sets the hEvent to be stimulated one. The calling program can determine the state of event object hEvent by using the wait functions (WaitForSingleObject() or WaitForMultipleObjects()). Then, the GetoverlappedResult() is called to determine whether the operation of the function WaitCommEvent() is ended. The variables pointed by parameter lpEvtMask of the function WaitCommEvent() indicate the occurring communication events.

For serial communication devices, the Win32 API can support synchronous and asynchronous I/O operation modes. The program design of the synchronous operation mode is relatively simple, but the $\mathrm{I} / \mathrm{O}$ read-write functions cannot be returned before ending the $\mathrm{I} / \mathrm{O}$ read and write operations, which will suspend the calling threads until ending the $\mathrm{I} / \mathrm{O}$ read and write operations. The asynchronous operation mode is also known as overlapped operation mode and is more complex. However, it can make time-consuming I/O operation be executed in the background, so that the calling threads cannot be suspended. In fact, the asynchronous I/O operation in the operation system is completed by using the thread technology. This is very effective to improve the response speed of the calling threads in the communication of large amount of data. Therefore, the asynchronous operation mode should be used in the efficient serial communication program.

\section{Conclusion}

The emotional stability test system changes the emotional stability test from qualitative to quantitative analysis. The system can quantitatively analyze the emotional stability parameters of testees and is not affected by human factors, showing objectiveness and accuracy. The system provides a guarantee for carrying out a wide range of psychological tests for the talent selection and obtaining the accurate test results with a uniform standard. This system combining software with hardware enriches the means of the psychological test, and greatly improves the scientificity, accuracy and operability of the test.

\section{Acknowledgement}

This work is the key research project of teaching reforms of higher education in Shaanxi province, China (No. 15BZ64) and supported by the special funds for education and teaching reform in Shaanxi province, China.

\section{References}

[1] Robert E. Lewis, Robert J. Heckman. Talent management: A critical review[J]. Human Resource Management Re-view, 2006 (16):139-154.

[2] Mao Ailin. Talent Management: New Researching Trend of HRM[J]. Management and Review of Social Sciences, 2012(04):56-61.

[3] CHEN Jia. New Normal of Talented Management Based on Business Operating[J]. Journal of Beijing University of Technology(Social Sciences Edition), 2015(03):21-25.

[4] LI Xuefu. Talent management software application will become the new normal for Chinese companies[J]. Human Resource Management, 2015(02):49-52. 
[5] PAN Qidong, ZEMG Mi. Designing research of coal mine emergency management information system based on data integration[J]. Journal of Henan Polytechnic University(Natural Science), 2011(05):521-524.

[6] AN Zhiyu, CHENG, Jinlin The Study on the Design of Performance Management System Based on BSC--A Case of Insurance Corporation[J]. East China Economic Management, 2005(06):102-104. 